\title{
Investigation on Mutual Contention Bandwidth Request Mechanisms in Two-Hop Relay Network with ITU-R Path Loss Models
}

\author{
Rajesh Anbazhagan and Nakkeeran Rangaswamy \\ Department of Electronics Engineering, School of Engineering and Technology, Pondicherry University, Puducherry 605 014, India
}

Correspondence should be addressed to Rajesh Anbazhagan; rajesh@pec.edu

Received 14 March 2013; Accepted 29 April 2013

Academic Editors: M. Miśkowicz, K. Teh, and A. M. Tonello

Copyright (c) 2013 R. Anbazhagan and N. Rangaswamy. This is an open access article distributed under the Creative Commons Attribution License, which permits unrestricted use, distribution, and reproduction in any medium, provided the original work is properly cited.

\begin{abstract}
The performance of two-hop contention based bandwidth request (BR) mechanism for WiMAX relay networks is investigated under ITU-R path loss models. In conventional WiMAX systems, the mobile stations (MS) update their contention window irrespective of their transmission failures. Those systems update their contention window on collision and due to channel error or unavailability of bandwidth. Further, these failure models have been suggested for single hop networks. The failure model in two-hop systems becomes complex since it may include additional failure events such as improper detection of codes and channel error due to varying path loss. Interestingly, these failure events (collision, channel error, unavailability of bandwidth, and improper detection of codes) do not occur evenly for both hops of a link. Hence, to set the contention window effectively, unique failure models are developed by considering the characteristics of BR mechanism and hop at which the BR is performed. In the proposed system, the two-hop BR is carried out with all combinations of message and code bandwidth request schemes. Among them, the messagecode BR mechanism performs better under suburban fixed and outdoor to indoor or pedestrian environment, and code-code BR scheme performs better for vehicular environment.
\end{abstract}

\section{Introduction}

Broadband wireless technologies with worldwide interoperability for microwave access (WiMAX) networks are gaining tremendous attention due to the increasing consumer demands. WiMAX is envisioned to provide high-speed data rate to last mile and last inch customers. In recent years, mobile WiMAX has emerged to support high-speed data rate to customers at vehicular speed. The IEEE 802.16j task group has been formed to extend the scope of single hop WiMAX network (IEEE 802.16e) in terms of capacity (throughput) and coverage [1]. In WiMAX relay network, the stations of interest are base station (BS), relay station (RS), and mobile station (MS). Based on the number of hops between BS and MS, the WiMAX networks may be classified into two types, namely, single hop network and multihop network. Further, the architecture modes supported by the WiMAX standard are as follows: point-to-point (PP), point-to-multipoint
(PMP), and mesh modes. In a single hop network, the MS communicate directly with the BS, and hence the network follows PP (between BSs) or PMP architecture $[2,3]$. In multihop network, the MS may not be in a position to communicate directly with the BS and involve multiple hops to establish the connectivity. One or more RS are involved to establish the connectivity between BS and MS, and the network follows either tree mode architecture. Nevertheless, relay based multihop WiMAX networks are envisioned as a promising solution for coverage extension, coverage hole, shadowing, and throughput enhancement [4-7].

The WiMAX relay network may be deployed in rural, urban, suburban, or at vehicular environment. The performance of WiMAX relay can vary significantly for the different environments due to differences in mobility pattern and physical signal propagation terrain. As in conventional wireless communication systems, the effect of path loss in WiMAX has to be considered while evaluating the network 
performance. The pathloss model reflecting the practical propagation scenarios remains challenging, which determines the actual network performance. Many works have been dedicated to designing effective techniques to overcome signal degradation due to the effect of pathloss, and WiMAX makes use of number of advanced techniques, such as orthogonal frequency division multiple access (OFDMA), adaptive modulation and coding (AMC), and multiple input multiple output (MIMO) antenna [8-10]. In this paper, the effects of contention mechanism due to various pathloss models have been investigated.

The contention based bandwidth request (BR) mechanisms in WiMAX networks are classified into message BR and code division multiple access (CDMA) or simply code BR [11]. There always exists a tradeoff between message and CDMA BR with single hop networks. The former suffers from low contention efficiency and high access delay amid low signaling overhead with increase in the number of stations. The latter performs well with high contention efficiency and low access delay whereas the signaling overhead is very high when compared to message based bandwidth request. Further, the performance of CDMA based BR reduces with increase in the number of codes (also with increase in the number of stations) allocated for BR. Although many works have been carried out with contention based BR in single hop networks, no work has been cited in the literature to evaluate the performance over two-hop WiMAX relay networks. As the pathloss between BS and MS is more diversified compared to the pathloss between MS and RS, the probability of successful bandwidth request depends on the choice of message or code bandwidth request over these hops of a link.

The remainder of the paper is structured as follows: the motivation towards contention based bandwidth request analysis with ITU-R pathloss model is discussed in Section 2. The works related to bandwidth request for WiMAX relay networks are detailed in Section 3. The proposed contention bandwidth request for WiMAX relay network is explained in Section 4, and their analysis with three pathloss models is presented in Section 5. The performance evaluation of the proposed bandwidth request mechanisms is analyzed in Section 6, and concluding remarks are given in Section 7.

\section{Motivation}

In mobile communication, the bit error rate or block error rate of the system increases as the distance between the transmitter and receiver increases due to pathloss experienced by the transmitted signal. The channel error due to pathloss may have significant effect on system performance as the probability of failure in contention based bandwidth request depends on the channel error. Other parameters that affect the contention efficiency include collision due to contention and unavailability of bandwidth. Further, the effect of pathloss on message based BR and code based BR may differ due to their unique signal processing mechanism. Nevertheless, the performance of contention based BR for multihop networks has to be investigated as the pathloss is a function of the distance between the transmitter and receiver.
TABLE 1: ITU-R pathloss model specification [31].

\begin{tabular}{lc}
\hline Parameters & Details \\
\hline Environment/scenarios & $\begin{array}{c}\text { Suburban fixed, vehicular, or outdoor } \\
\text { to indoor and pedestrian } \\
\text { Multipath channel }\end{array}$ \\
Frequency range & $2-6 \mathrm{GHz}$ \\
Maximum bandwidth & $100 \mathrm{MHz}$ \\
Mobility & $\mathrm{Up} \mathrm{to} 365 \mathrm{~km} / \mathrm{h}$ \\
Delay spread & $20 \mathrm{~ns}-370 \mathrm{~ns}$ \\
BS angle spread & $6^{\circ}-42^{\circ}$ \\
MS angle spread & $30^{\circ}-74^{\circ}$ \\
\hline
\end{tabular}

Since the practical environment was too complex to model, many pathloss models have been developed considering the frequency of operation, mobility of stations, application, the type of environment, and the system under consideration. The pathloss models developed so far have been in accordance with the specification governed by either ITU or 3GPP [12]. Any transmission technique or algorithm is effective only if it is tested under these pathloss models. Further, one can also design techniques by exploiting the properties of the pathloss model. In early days pathloss models such as Okumura-Hata model, Lee model, and European Cooperation in Science and Technology (COST) 231 model and so forth have been developed for narrowband systems. The authors in [13] have analyzed the possibility of using COST 231, Erceg, SUI, and ECC propagation models for WiMAX coverage planning that is developed primarily for GSM and UMTS. However, with increase in data rate the narrowband models have been enhanced by considering the delay spread and resulted in wideband models such as ITU-R (spatial channel model (SCM)) and COST 207.

Among the wideband models, the ITU-R specifies the experiential model for WiMAX network. Accordingly, the pathloss models have been categorized into suburban fixed, vehicular environment and outdoor to indoor/pedestrian environment. Since the delay spread varies significantly based on the environment, the ITU-R specifies two delay spread for each environment, namely, low delay spread (channel A) and medium delay spread (channel B). The specification made by ITU-R is shown in Table 1. Environment or scenario specific investigation has to be made as the performance of WiMAX relay network may vary significantly due to the differences in physical signal propagation terrain. Further, the other factors such as traffic condition, frequency of operation $(2.4 \mathrm{GHz}$, $3.2 \mathrm{GHz}$, or $5.8 \mathrm{GHz}$ ), and density and mobility of stations have significant impact on the contention performance of multihop WiMAX networks [14-16].

Although, many research attempts have been carried out in evaluating the performance of different transmission technologies, signal processing techniques, and receiver algorithms for WiMAX networks under various pathloss models, no effort has been carried out with multistage contention based BR in WiMAX relay network. Hence, the focus of this paper is to investigate the performance of contention based BR under different pathloss models including suburban fixed, 
outdoor to indoor/pedestrian and vehicular environment and suggest an appropriate bandwidth request mechanisms based on the environment.

\section{Related Works}

In the literature, many works have been proposed for relaybased networks to enhance the system performance. In this section, we review the works related to relay based WiMAX networks and the pathloss models associated with WiMAX networks. So [17] has analyzed the impact of signaling overhead of mobile application part (MAP) messages with voice over internet protocol (VOIP) services for uplink and downlink transmissions in IEEE 802.16e systems. Fath Elrahman Ismael et al. [18] have considered CDMA based contention resolution for bandwidth request in mobile multihop relay networks and proposed grey prediction algorithm (GPA) to reduce the delay associated with bandwidth allocation. Upase and Hunukumbure [19] have made a detailed discussion on the cost and signaling analysis related to IEEE802.16j networks and provided the economic possibility of deploying the WiMAX networks with relay stations. Chu and Huang [20] have considered consecutive bandwidth request schemes (CBRS) to overcome collisions due to contention when bandwidth request by relay stations is made with contention based bandwidth request. Niyato et al. [21] have considered relay centric radio resource management and formulated chance constrained assignment problem (CCAP) to optimize the amount of bandwidth reserved for relay station.

Mach and Bestak [22] have analyzed the performance (throughput and packet delay) of distributed relays for resource allocation with unsolicited grant service (UGS), real time polling service (rtPS), and nonreal time polling service (nrtPS). Becvar and $\mathrm{MacH}$ [23] have designed a reporting technique with minimum management overhead for monitoring the signal quality when mobile stations move between base station and relay stations. Zhang et al. [24] have considered a dedicated resource allocation or extra resource reservation (ERR) at relay stations to reduce service flow management signaling overhead, since the relay stations exhibit additional overhead when compared to nonrelay based network.

Mai et al. [25] have proposed zone based bandwidth allocation (ZBA) for relay stations in which the bandwidth is allocated to relay stations based on the probability of users that visit the relay stations. However, the ERR and ZBA can be considered only when excess of resource is available at the BS and with lesser number of hops between BS and MS. The characterization of network resource optimization in relayassisted network under practical constraints is illustrated in [26]. The performance of the network is evaluated assuming the availability and quality of the channel state information (CSI) at the BS, MS, and RS. In particular, the transmitter knows the pathloss. However, frequent exchange of CSI between MS to RS and RS to BS introduces additional overhead in the network.

Although many works [17-26] have been proposed to increase the throughput and extend the coverage with relay based networks, the target performance is achieved with either additional resource (bandwidth) at RS. Further, no works have been proposed to suggest an appropriate multihop contention mechanism in WiMAX relay network based on the environment. Therefore, in this paper, a heuristic contention based bandwidth request mechanisms are proposed with the motivation of reducing the connection and queuing delay with improved network throughput.

\section{Contention Bandwidth Request for WiMAX Two-Hop Relay Network}

The introduction of relay stations significantly modifies the topology of conventional WiMAX systems and increases the complexity of resource (bandwidth) allocation in the system. Though static bandwidth assignment (polling between MS and RS) at the RS is preferred, the network suffers from insufficient bandwidth to MS with increased and dynamic placement of relay stations. The possible solution is the contention based bandwidth request at RS. With contention based BR, there is a need for heuristic bandwidth request design to meet the QoS requirements of individual end user.

This section outlines the proposed BR mechanisms for WiMAX relay networks. Although the relay considered in this paper is configured with nontransparent mode, the BS alone can grant the bandwidth request. Further, the BS does not distinguish RS from MS. Hence, the BR from RS is the same as that from MS to BS. Further, the MS also does not distinguish between BS and RS. The BR from MS with RS is executed in the similar way as that with BS. Although the WiMAX network can contain multiple hops, we consider only two hops (single relay) between BS and MS as shown in Figure 1. The hop1 represents the air interface between MS to RS and hop2 represents the air interface between RS to BS. With two-hop networks, there arise four possible combinations for bandwidth request between MS to RS and RS to BS.

In message-message ( $\mathrm{MM}$ ) based contention resolution, the MS contends for transmission opportunity to perform bandwidth request. The transmission opportunity is randomly selected from the available slots for bandwidth request that are broadcasted by the BS. This selected random value indicates the number of contention transmission opportunities that the MS has to defer before transmitting. If the MS is successful in obtaining the transmission opportunity, then it proceeds in transmitting the bandwidth request. If unsuccessful, it undergoes contention resolution with truncated binary exponential backoff (TBEB). On successful bandwidth request from MS, RS performs the similar bandwidth request as executed by the MS. Since the network is configured with centralized scheduling, the probability of success in WiMAX depends only on the BS having enough bandwidth to accept the request. If the bandwidth request is successful, BS provides the bandwidth grant to the RS that is then notified to the MS.

In code-code (CC) based contention resolution, BS defines the ranging subchannel and subset of ranging codes. The WiMAX standard defines 256 codes for initial ranging, 

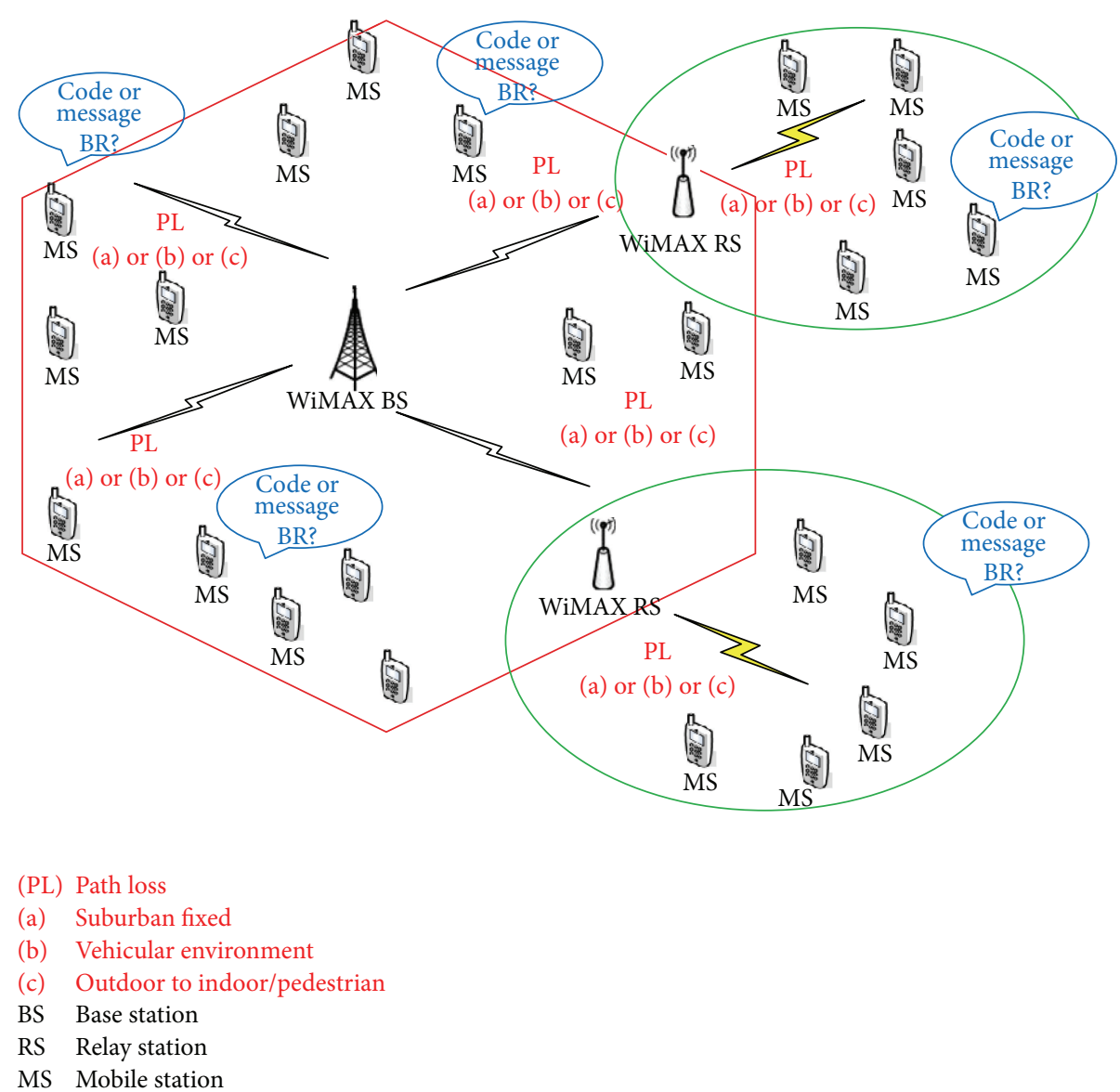

FIGURE 1: WiMAX two-hop relay network for contention based bandwidth request mechanism.

periodic ranging, handoff ranging, and bandwidth request. The BS defines a subset of codes from 256 to be used by the MS for bandwidth request. The MS, which is requiring bandwidth randomly, selects a ranging subchannel and a ranging code from the subset allocated and transmits the bandwidth request to the RS. After receiving the request, RS decodes the request, sends a new request by randomly selecting a ranging subchannel and a ranging code from its subset, and transmits the bandwidth request to the BS. On receiving the request from RS, BS broadcasts CDMA_ALL_IE (CDMA allocation information element) message. The CDMA_ALL_IE contains the bandwidth request code, the frame number, the symbol number, and the subchannel number to be used by the RS for transmitting the code based bandwidth request. The corresponding RS will decode the information by comparing with its ranging request information and retransmits it to the MS.

Then, the MS uses the allocation (information element) to send bandwidth request to the BS via RS. On receiving the request, the BS will allocate the uplink bandwidth. Unlike in message based bandwidth request, the MS with code based method can make use of different CDMA codes through one ranging channel and executes the bandwidth request mechanism with fewer resources. However, the collision occurs in code based method if the code and randomly selected TxOP are the same among two or more CDMA packets. If MS is unsuccessful in transmitting the bandwidth request, it undergoes TBEB mechanism. An efficient design of contention resolution improves the probability of transmission but the probability of success in relay based WiMAX depends only bandwidth request on being successful on both hops. Though the MM based bandwidth request is simple in its operation, it undergoes high probability of collision (during hop2) due to overlapping of backoff counters with increase in the number of contending stations (MSs that contend for resource with RSs). Therefore, the probability that the MSs transmit their bandwidth request over the same transmission opportunity is high with MM based BR.

Similarly, the probability of collision with CC based BR is less (with lesser number stations) when compared to message based bandwidth request; it suffers from large signaling overhead. Nevertheless, with a maximum of 64 CDMA codes allocated for bandwidth request (out of 256), the contention efficiency and access delay decrease with increase in the number of ranging codes. Nevertheless, the contention resolution with CC based bandwidth request suffers from transmission failure due to the detection threshold at the RS. The detection threshold has to be chosen as an optimum value since larger value of detection threshold increases the probability of miss and smaller value increases the probability of false detection. 
However, the detection threshold at the RS is fixed and it does not vary according to the channel conditions. This makes the performance of code based bandwidth request at hopl less significant with relay based bandwidth request. Further, the code based BR results in more frame delay with its bandwidth request mechanism. Hence, the message based bandwidth request is more appropriate between MS and RS. However, the bandwidth request between RS and BS is CDMA based, since the BS tunes its detection threshold based on the current state of the channel. Therefore, we suggest a heuristic BR to improve the network throughput while reducing connection delay without any dedicated bandwidth allocation at RS.

The operation of message-code (MC) BR is as follows. The MS randomly selects the TxOP and transmits the bandwidth request to the RS. If the transmission is unsuccessful, it undergoes contention resolution with truncated binary exponential backoff (TBEB). The backoff mechanism is executed to avoid overlapping of backoff counters between the MSs else it results in collision. Further, RS does not send any acknowledgement on decoding the bandwidth request. If MS receives a ranging response (RNG-RSP) message after the contention transmission, the contention resolution is completed. If no such message is received before "T16" (waiting time or response time) expires, the MS may regard the transmission as lost. The waiting time is the time over which the MS waits for response from RS after executing the bandwidth request.

If the request is successful, then RS randomly selects a ranging subchannel and a ranging code from the subset allocated by BS and transmits the bandwidth request to the BS. On receiving the request, the BS broadcasts CDMA_ALL_IE message. The corresponding RS will decode the information and send the response to the MS. The MS then transmits the bandwidth request to the RS that is relayed to the BS via RS. On receiving the request, the BS allocates the requested bandwidth to the RS which is then relayed to the MS. Apart from MC based BR mechanism, we have also investigated the $\mathrm{CM}$ based $\mathrm{BR}$ where code based $\mathrm{BR}$ is carried out between MS and RS and message based BR is performed between RS and MS. The operation of code message (CM) based BR is reverse to the mechanism discussed with MC based BR.

\section{Analysis of Successful Bandwidth Request Mechanisms under ITU-R Pathloss Models}

Although a heuristic means of bandwidth request mechanisms is being attempted for three different environments, only a selective mechanism performs better among the four possible options of collaborative bandwidth request. With suburban fixed or indoor office environment and outdoor to indoor/pedestrian $(\mathrm{OI} \& P)$ environment, $\mathrm{MC}$ bandwidth request performs better than other three BR mechanisms. In case of vehicular environment, $\mathrm{CC}$ bandwidth request performs better than other three BR mechanisms. Hence, we proceed further in deriving the contention efficiency and access delay of $\mathrm{MC}$ and CC BR mechanisms.

In WiMAX systems, many methods have been suggested for updating and controlling the size of contention window of MSs [27-30]. However, these methods consider the probability of failure of three possible events, namely, collisions due to contention, unavailability of bandwidth, and channel error. Ni et al. [28] modeled the probability of failure $\left(P_{f}\right)$ with collision and channel error. According to them, $P_{f}$ is given by

$$
P_{f}=P_{c}+P_{e}-P_{c} P_{e},
$$

where $P_{e}$ denotes the probability of error due to channel and $P_{c}$ denotes the probability of collision due to contention. Fallah et al. [27] and Chuck et al. [29] modeled the probability of failure with the collision and unavailability of bandwidth, which is formulated by

$$
P_{f}=P_{c}+\left(1-P_{c}\right)(1-q)^{T_{r}},
$$

where $q$ denotes the probability of BS to accept a bandwidth request and $T_{r}$ denotes response time or waiting time. However, the transmission failure model has not been modeled with the above said three possible events. Further, the system suffers from improper detection of CDMA codes when code BR is carried out at RSs. Nevertheless, varying pathloss introduces additional failure at MS. Setting of contention window at MS will be effective if the model appropriately incorporates the aforementioned failure events. Further, the occurrence of these failure events is not uniform in hopl and hop2 of the link. By defining the failure model at two hops appropriately, the MSs will update their contention window accurately. Such a complex model for setting the contention window is required since improper value of contention window will reduce the contention efficiency and increase the access delay amid MSs.

5.1. Contention Efficiency and Access Delay for Suburban Fixed or Indoor Office Environment. The probability of failure at two hops of the link between MS and BS via RS is assumed to be independent. If $P_{f 1}$ represents the probability of failure at hop1 and $P_{f 2}$ signifies the probability of failure at hop2, then the total probability of failure $\left(P_{f}\right)$ can be formulated as follows:

$$
P_{f}=1-\left(\left(1-P_{f 1}\right)\left(1-P_{f 2}\right)\right) .
$$

Since message bandwidth request is carried out at hopl, the probability of failure $\left(P_{f 1}\right)$ could be either due to collision or channel error and is given as follows:

$$
P_{f 1}=1-\left(\left(1-P_{c 1}\right)\left(1-P_{e 1}\right)\right),
$$

where $P_{c 1}$ refers to the probability of failure as a result of collision due to contention at hop1 and $P_{e 1}$ refers to the probability of failure due to channel error. The backoff time in contention resolution process is set to follow geometric distribution. Hence, the number of failures until the first success is given as follows:

$$
P(W=k)=(1-p)^{k} p, \quad 0 \leq k \leq \infty .
$$


The probability of transmission $\left(p_{1}\right)$ by a given station at hopl can be computed by estimating the mean of the contention window $(W)$ and is derived as follows:

$$
E[W]=\sum_{i=0}^{m} W_{i} p\left(W_{i}\right)
$$

where $m$ is the maximum backoff stage, and solving (6) further, it becomes

$$
E[W]=W \frac{\left(1-P_{f 1}\right)\left(1-\left(2 P_{f 1}\right)^{m+1}\right)}{\left(1-\left(2 P_{f 1}\right)\right)\left(1-P_{f 1}^{m+1}\right)} .
$$

The relation between the probability of transmission $\left(p_{1}\right)$ at hopl and mean of contention window is given as follows:

$$
E[W]=\frac{1-p_{1}}{p_{1}} .
$$

Equating (7) and (8) and solving for $p_{1}$ result in

$$
p_{1}=\frac{\left(1-P_{f 1}^{m+1}\right)\left(1-\left(2 P_{f 1}\right)\right)}{W\left(1-P_{f 1}\right)\left(1-\left(2 P_{f 1}\right)^{m+1}\right)+\left(1-\left(2 P_{f 1}\right)\right)\left(1-P_{f 1}^{m+1}\right)} .
$$

The conditional collision probability $\left(P_{c 1}\right)$ at hopl is given as follows:

$$
P_{c 1}=1-\left(1-p_{1}\right)^{\left(n_{1} / r_{1}\right)-1}
$$

where $r_{1}$ represents the number of RSs at hopl and $n_{1}$ represents the number of MSs at hopl.

The pathloss between MS and RS at hopl over suburban fixed environment is given as follows [31]:

$$
\mathrm{PL}_{\mathrm{RS}-\mathrm{MS}}(\mathrm{dB})=30 \log _{10}\left(d_{1}\right)+18.3 n^{((n+2) /(n+1)-0.46)}+37,
$$

where $d_{1}$ is the distance between the RS and MS at hop1 and $n$ is the number of floors in the path. The probability of error at hopl is given by:

$$
P_{e 1}=f n\left(\text { Channel_Noise } \text { hop1 }_{1}, \mathrm{PL}_{\mathrm{RS}-\mathrm{MS}}\right) \text {. }
$$

The contention efficiency is defined as the product of probability of transmission of bandwidth request by the stations and the probability of successful transmission. Thus, the contention efficiency at hop1 $\left(\eta_{1}\right)$ is derived by using conditional probability over three events, namely, the probability of successful bandwidth request given that the request is not affected by channel error and failure due to detection of transmitted CDMA code at hopl. Hence, $\eta_{1}$ is given as follows:

$$
\eta_{1}=\frac{n_{1} p_{1}}{r_{1}}\left(1-p_{1}\right)^{\left(n_{1} / r_{1}\right)-1}\left(1-P_{e 1}\right)
$$

The access delay $\left(D_{1}\right)$ with a given number of transmission opportunities $\left(T_{O}\right)$ available is defined as the ratio of the number of stations at hopl to the product of contention efficiency and $T_{O}[27]$ and is given as follows:

$$
D_{1}=\frac{n_{1}}{r_{1} T_{O} \eta_{1}} .
$$

With code bandwidth request carried out at hop2, the probability of failure $\left(P_{f 2}\right)$ is because of probability of collision due to contention $\left(P_{c 2}\right)$ at hop2, probability of channel error $\left(P_{e 2}\right)$ at hop2, probability of failure due to detection of transmitted CDMA code $\left(P_{f_{c 2}}\right)$ at hop2, and probability of unavailability of bandwidth $\left(P_{u 2}\right)$ at hop2. Hence, $P_{f 2}$ is given as follows:

$$
P_{f 2}=1-\left(\left(1-P_{c 2}\right)\left(1-P_{e 2}\right)\left(1-P_{f c 2}\right)\left(1-P_{u 2}\right)\right) .
$$

The conditional collision probability $\left(P_{c 3}\right)$ at hop3 is given as follows:

$$
P_{c 2}=1-\left(1-\left(p_{2} / R_{2}\right)\right)^{n_{2}-1}
$$

where $R_{2}$ represents the number of codes available for bandwidth request at hop 2 and $n_{2}$ represents the number of mobile stations at hop2.

The pathloss between RS and BS at hop2 over suburban fixed environment is given as follows [31]:

$$
\mathrm{PL}_{\mathrm{BS}-\mathrm{RS}}(\mathrm{dB})=30 \log _{10}\left(d_{2}\right)+18.3 n^{((n+2) /(n+1)-0.46)}+37,
$$

where $d_{2}$ is the distance between the BS and RS at hopl and $n$ is the number of floors in the path. The probability of error at hop1 is given by

$$
P_{e 2}=f n\left(\text { Channel_Noise } \mathrm{hop}_{2}, \mathrm{PL}_{\mathrm{BS}-\mathrm{RS}}\right) \text {. }
$$

The probability of failure due to detection of transmitted CDMA code $\left(P_{f c 2}\right)$ at hop2 is given as follows:

$$
P_{f c 2}=\sum_{i=1}^{0.5\left(T_{2}+144(C-2)\right)}\left(\begin{array}{c}
144(C-1) \\
i
\end{array}\right) 0.5^{144(C-1)},
$$

where 144 refers to the number of subcarriers, $T_{2}$ is the detection threshold at hop2, and $C$ is the number of codes transmitted on the ranging channel.

The probability of unavailability of bandwidth $\left(P_{u 2}\right)$ at hop2 is given as follows:

$$
P_{u 2}=\left(1-P_{c 2}\right)\left(1-P_{e 2}\right)\left(1-P_{f c 2}\right)(1-q)^{T_{r}} .
$$

The contention efficiency at hop2 $\left(\eta_{2}\right)$ is derived by using conditional probability over four events, namely, the probability of successful bandwidth request given that the request is not affected by channel error, failure due to detection of transmitted CDMA code, and availability of bandwidth at the BS. Hence, $\eta_{2}$ is given as follows:

$$
\begin{aligned}
\eta_{2}= & n_{2} p_{2}\left(1-\left(\frac{p_{2}}{R_{2}}\right)\right)^{n_{2}-1}\left(1-P_{e 2}\right)\left(1-P_{f c 2}\right) \\
& \times\left(1-(1-q)^{T_{r}}\right) .
\end{aligned}
$$


The access delay $\left(D_{2}\right)$ with a given number of transmission opportunity $\left(T_{O}\right)$ available at hop2 is given as follows:

$$
D_{2}=\frac{n_{2}}{T_{O} \eta_{2}}
$$

The total contention efficiency under suburban fixed $\left(\eta_{\text {Suburban_Fixed }}\right)$ environment is given by

$$
\eta=\eta_{1} \times \eta_{2}
$$

Substituting (15), (20), and (27) in (29), $\eta_{\text {Suburban_Fixed }}$ is given as follows:

$$
\begin{aligned}
\eta_{\text {Suburban_Fixed }}= & \frac{n_{1} p_{1}}{r_{1}}\left(1-p_{1}\right)^{\left(n_{1} / r_{1}\right)-1}\left(1-P_{e 1}\right) \\
& \times n_{2} p_{2}\left(1-\left(\frac{p_{2}}{R_{2}}\right)\right)^{n_{2}-1}\left(1-P_{e 2}\right) \\
& \times\left(1-P_{f c 2}\right)\left(1-(1-q)^{T_{r}}\right), \\
\eta_{\text {Suburban_Fixed }}= & \left(\frac{n_{1} n_{2}}{r_{1}}\right)\left(p_{1} p_{2}\right)\left(\left(1-P_{e 1}\right)\left(1-P_{e 2}\right)\right) \\
& \times\left(1-P_{f c 2}\right)\left(1-p_{1}\right)^{\left(n_{1} / r_{1}\right)-1} \\
& \times\left(1-\left(\frac{p_{2}}{R_{2}}\right)\right)^{n_{2}-1}\left(1-(1-q)^{T_{r}}\right) .
\end{aligned}
$$

The total access delay under suburban fixed $\left(D_{\text {Suburban_Fixed }}\right)$ environment is given by

$$
D_{\text {Suburban_Fixed }}=D_{1}+D_{2} \text {. }
$$
follows:

Substituting (16), (21), and (28) in (31), D is given as

$$
\begin{gathered}
D_{\text {Suburban_Fixed }}=\frac{n_{1}}{r_{1} T_{O} \eta_{1}}+\frac{n_{2}}{T_{O} \eta_{2}}, \\
D_{\text {Suburban_Fixed }}=\frac{1}{T_{O}}\left(\frac{n_{1} \eta_{2}+n_{2} r_{1} \eta_{1}}{r_{1} \eta_{1} \eta_{2}}\right) .
\end{gathered}
$$

The contention efficiency and the access delay with MC $\mathrm{BR}$ increase with reduction in the number of codes assigned for bandwidth request. In this case, the total probability of failure mainly depends on the improper detection of codes and channel error rather than all events. Since the delay spread with suburban fixed is relatively low than other environments under consideration, MC bandwidth request performs better than other three bandwidth request mechanisms.

5.2. Contention Efficiency and Access Delay for Vehicular Environment. The derivations with $\mathrm{MC}$ can be extended to derive the contention efficiency and access delay with CC bandwidth request. The variations are with the probability of error and hence its pathloss component associated with vehicular environment. The pathloss between MS and RS at hop1 over vehicular environment is given as follows [31]:

$$
\begin{aligned}
\mathrm{PL}_{\mathrm{RS}-\mathrm{MS}}(\mathrm{dB})= & 40\left(1-\left(0.004 \times \delta h_{r}\right)\right) \log _{10}\left(d_{1}\right) \\
& -18 \log _{10}\left(\delta h_{r}\right)+21 \log _{10}(f)+80,
\end{aligned}
$$

where $\delta h_{r}$ is the difference between RS antenna height and the building height, $d_{1}$ is the distance between the RS and MS at hopl, and $f$ is the carrier frequency. With a carrier frequency of $5.8 \mathrm{GHz}$ and $\delta h_{r}$ of $15 \mathrm{~m}$, the pathloss at hopl simplifies to

$$
\begin{gathered}
\mathrm{PL}_{\mathrm{RS}-\mathrm{MS}}(\mathrm{dB})=31.6 \log _{10}\left(d_{1}\right)+31.82, \\
P_{e 1}=f n\left(\text { Channel_Noise } \mathrm{hopl}_{\text {ho }}, \mathrm{PL}_{\mathrm{RS}-\mathrm{MS}}\right) .
\end{gathered}
$$

The pathloss between RS and BS at hop2 over vehicular environment is given as follows [31]:

$$
\begin{aligned}
\mathrm{PL}_{\mathrm{BS}-\mathrm{RS}}(\mathrm{dB})= & 40\left(1-\left(0.004 \times \delta h_{b}\right)\right) \log _{10}\left(d_{2}\right) \\
& -18 \log _{10}\left(\delta h_{b}\right)+21 \log _{10}(f)+80,
\end{aligned}
$$

where $\delta h_{b}$ is the difference between BS antenna height and the building height and $d_{2}$ is the distance between the RS and BS at hop2. With a carrier frequency of $5.8 \mathrm{GHz}$ and $\delta h_{b}$ of $30 \mathrm{~m}$, the pathloss at hop 2 is given as follows:

$$
\begin{gathered}
\mathrm{PL}_{\mathrm{BS}-\mathrm{RS}}(\mathrm{dB})=35.2 \log _{10}\left(d_{1}\right)+26.4, \\
P_{e 2}=f n\left(\text { Channel_Noise } \text { hop } 2, \mathrm{PL}_{\mathrm{BS}-\mathrm{RS}}\right) .
\end{gathered}
$$

The total contention efficiency under vehicular $\left(\eta_{\text {Vehicular }}\right)$ environment is given by

$$
\begin{aligned}
\eta_{\text {Vehicular }}= & \left(\frac{n_{1} n_{2}}{r_{1}}\right)\left(p_{1} p_{2}\right)\left(\left(1-P_{e 1}\right)\left(1-P_{e 2}\right)\right) \\
& \times\left(\left(1-P_{f c 1}\right)\left(1-P_{f c 2}\right)\right)\left(1-\left(\frac{p_{1}}{R_{1}}\right)\right)^{\left(n_{1} / r_{1}\right)-1} \\
& \times\left(1-\left(\frac{p_{2}}{R_{2}}\right)\right)^{n_{2}-1}\left(1-(1-q)^{T_{r}}\right)
\end{aligned}
$$

The total access delay under vehicular $\left(D_{\text {Vehicular }}\right)$ environment is given by

$$
D_{\text {Vehicular }}=\frac{1}{T_{O}}\left(\frac{n_{1} \eta_{2}+n_{2} r_{1} \eta_{1}}{r_{1} \eta_{1} \eta_{2}}\right) .
$$

Comparing (24) and (33), one can infer that the vehicular contention efficiency reduces with increase in the number of codes $\left(r_{1}\right.$ and $\left.r_{2}\right)$ assigned for bandwidth request. However, with increase in delay spread, the foremost probability of failure is because of collision due to contention than with detection of codes and unavailability of bandwidth. Hence, $\mathrm{CC}$ bandwidth request performs better in vehicular environment. 
TABLE 2: Simulation parameters.

\begin{tabular}{|c|c|}
\hline Parameters & Details \\
\hline Multiple access & OFDMA \\
\hline Duplexing & TDD \\
\hline Frequency $(\mathrm{GHz})$ & 5.8 \\
\hline Frame duration (ms) & 5 \\
\hline Symbol duration (us) & 100.8 \\
\hline Number of subcarriers & 2048 \\
\hline Number of subchannels & 70 \\
\hline Usage mode & PUSC \\
\hline $\begin{array}{l}\text { Uplink subframe size } \\
\text { (symbols) }\end{array}$ & 12 \\
\hline Number of BS/RS/MS & $1 / 3 / 75$ \\
\hline BS coverage $(\mathrm{km})$ & 5 \\
\hline RS coverage $(\mathrm{m})$ & 300 \\
\hline Maximum hops & 2 \\
\hline Pathloss model & $\begin{array}{l}\text { Suburban fixed, vehicular, or } \\
\text { outdoor to indoor and pedestrian }\end{array}$ \\
\hline Multipath channel model & ITU Channel A \\
\hline Ranging power step (mW) & 0.25 \\
\hline $\begin{array}{l}\text { Bandwidth request backoff } \\
\text { start }\end{array}$ & 2 \\
\hline $\begin{array}{l}\text { Bandwidth request backoff } \\
\text { end }\end{array}$ & 4 \\
\hline $\begin{array}{l}\text { Reservation timeout } \\
\text { (contention) }\end{array}$ & 16 \\
\hline Request retries & 16 \\
\hline Application & Best effort (FTP and HTTP) \\
\hline Efficiency mode & Physical layer enabled \\
\hline Simulation platform & OPNET 14.5 \\
\hline
\end{tabular}

5.3. Contention Efficiency and Access Delay for Outdoor to Indoor and Pedestrian (OI\&P) Environment. Since MC bandwidth request performs better under outdoor to indoor and pedestrian environment, the contention efficiency and access delay can be formulated from the pathloss component of outdoor to indoor and pedestrian environment. The pathloss between MS and RS at hop1 over outdoor to indoor and pedestrian environment is given as follows [31]:

$$
\mathrm{PL}_{\mathrm{RS}-\mathrm{MS}}(\mathrm{dB})=40 \log _{10}\left(d_{1}\right)+30 \log _{10}(f)+49
$$

where $d_{1}$ is the distance between the RS and MS at hopl and $f$ is the carrier frequency. With a carrier frequency of $5.8 \mathrm{GHz}$, the pathloss at hopl simplifies to

$$
\begin{gathered}
\mathrm{PL}_{\mathrm{RS}-\mathrm{MS}}(\mathrm{dB})=40 \log _{10}\left(d_{1}\right)+47.71, \\
P_{e 1}=f n\left(\text { Channel_Noise }{ }_{\mathrm{hop} 1}, \mathrm{PL}_{\mathrm{RS}-\mathrm{MS}}\right) .
\end{gathered}
$$

The pathloss between RS and BS at hop2 is given as follows [31]:

$$
\mathrm{PL}_{\mathrm{BS}-\mathrm{RS}}(\mathrm{dB})=40 \log _{10}\left(d_{2}\right)+47.71,
$$

where $d_{2}$ is the distance between the RS and BS at hop2. The probability of error at hop 2 is obtained by

$$
P_{e 2}=f n\left(\text { Channel_Noise } \text { hop2 }_{2}, \mathrm{PL}_{\mathrm{BS}-\mathrm{RS}}\right) \text {. }
$$

The total contention efficiency under outdoor to indoor and pedestrian $\left(\eta_{\mathrm{OI} \& P}\right)$ environment is formulated as follows:

$$
\begin{aligned}
\eta_{\text {OI\&P }}= & \left(\frac{n_{1} n_{2}}{r_{1}}\right)\left(p_{1} p_{2}\right)\left(\left(1-P_{e 1}\right)\left(1-P_{e 2}\right)\right)\left(1-P_{f c 2}\right) \\
& \times\left(1-p_{1}\right)^{\left(n_{1} / r_{1}\right)-1}\left(1-\left(\frac{p_{2}}{R_{2}}\right)\right)^{n_{2}-1}\left(1-(1-q)^{T_{r}}\right) .
\end{aligned}
$$

The total access delay under outdoor to indoor and pedestrian $\left(D_{\mathrm{OI \& P}}\right)$ environment is given by

$$
D_{\mathrm{OI} \& P}=\frac{1}{T_{\mathrm{O}}}\left(\frac{n_{1} \eta_{2}+n_{2} r_{1} \eta_{1}}{r_{1} \eta_{1} \eta_{2}}\right) .
$$

Comparing (24) and (39), one could infer that the variables, namely, the availability of bandwidth $(q)$, the number of codes available for bandwidth request $\left(R_{2}\right)$, number of mobile stations $\left(n_{1}\right.$ and $\left.n_{2}\right)$, and relay stations $\left(r_{2}\right)$, affecting the contention efficiency remain the same. However, the main contribution to contention efficiency and access delay is from $P_{e 1}$ and $P_{e 2}$ that in turn depends on the pathloss of the environment.

\section{Simulation Results}

To evaluate the performance of the WiMAX relay system under three pathloss models, numerous end-to-end simulations are conducted. The OPNET 14.5 simulator is used for evaluation. The simulations are carried out by making suitable modification to the contention based bandwidth request process at the MAC layer. The configuration parameters used for the simulation are listed in Table 2. The simulated WiMAX two-hop relay network with a single BS, 3 RS and 75 MS is shown in Figure 2. It should be noted that the increase in simulation time is in conjunction with the increase in the number of stations that participate in the bandwidth request mechanism. Further, it is observed that almost all the stations participate when the simulation time reaches $300 \mathrm{~s}$. Therefore, to validate the effectiveness of the proposed mechanism, the simulation is extended to $600 \mathrm{~s}$. In addition, it should be noted that the contention efficiency and access delay are obtained in terms of throughput and connection delay, respectively, in the following subsections.

6.1. Simulation Platform. The applications considered in this paper with best effort (BE) services are heavy loaded hypertext transfer protocol (HTTP) and file transfer protocol (FTP). The nodes in the network are configured with FTP and heavily loaded HTTP model. FTP and HTTP traffic increases the data rate up to channel capacity by congestion control mechanism. To support best effort traffic, the start time offset has been set to uniform distribution with minimum outcome 


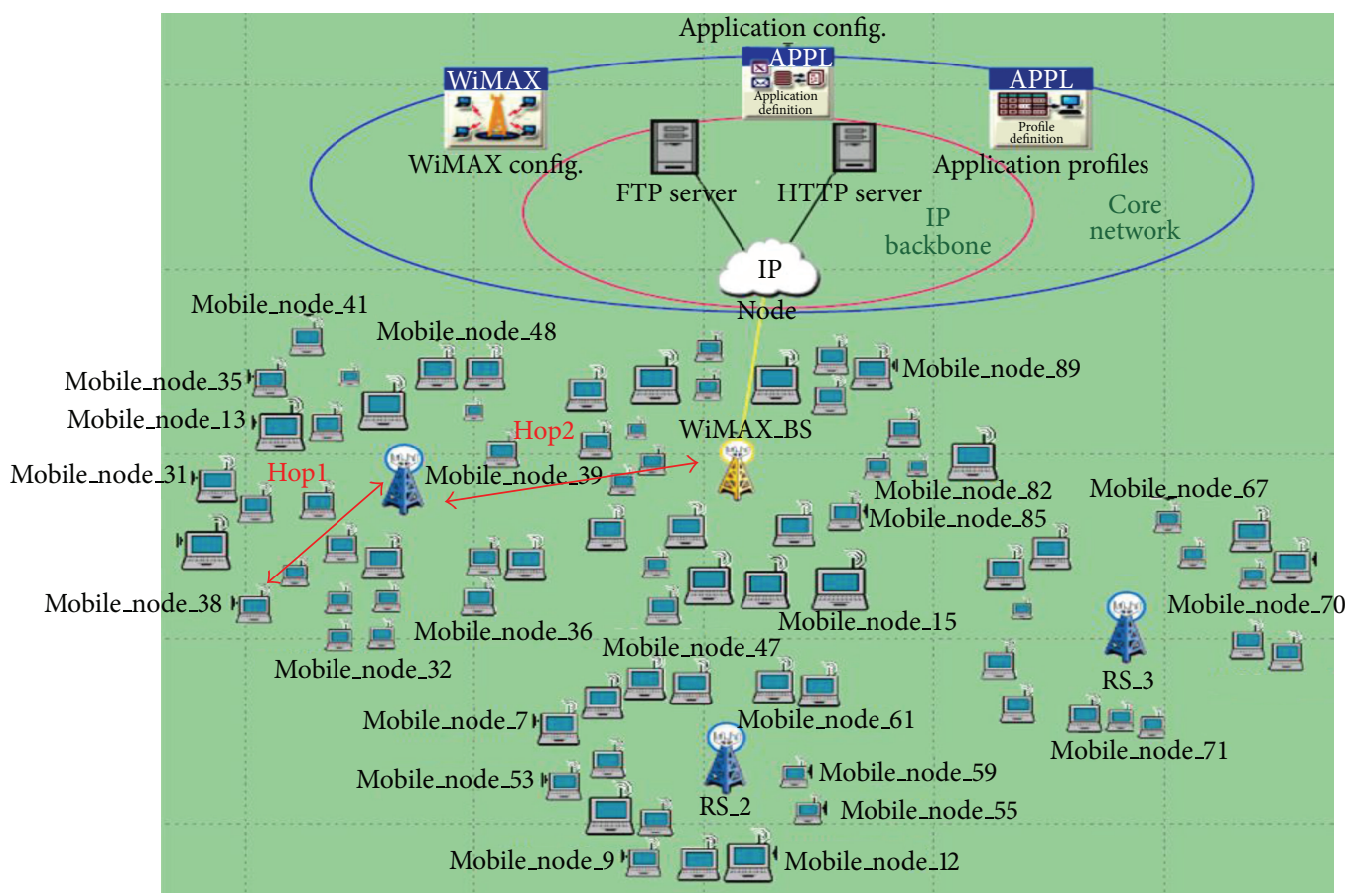

FIGURE 2: Simulation scenario for the proposed contention based bandwidth request mechanism.

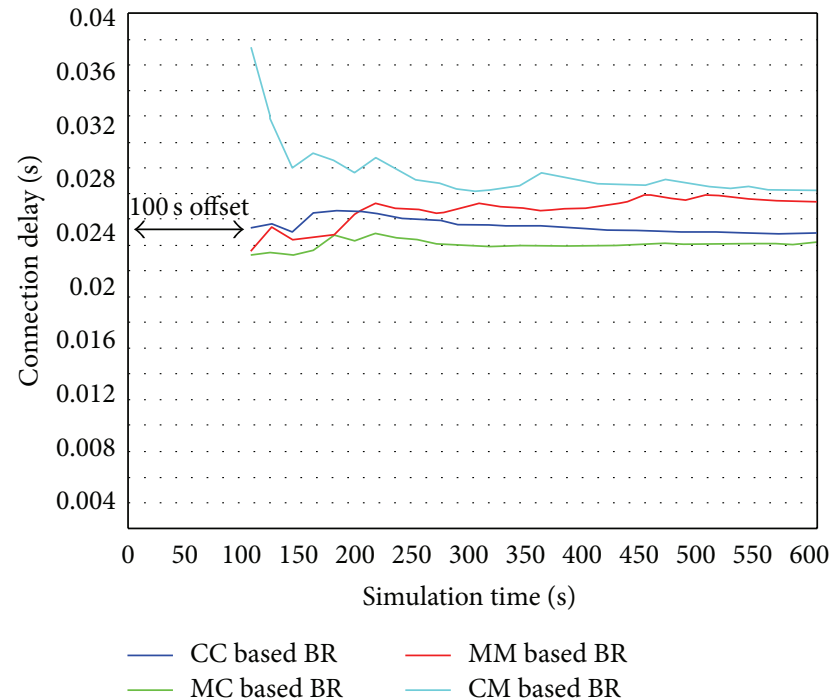

FIGURE 3: Connection delay as a function of simulation period for suburban fixed pathloss model.

of 50 and maximum outcome of 150 . Since the start time of FTP and HTTP application has been randomly chosen between minimum and maximum outcomes, the plot for BLER, uplink packets dropped, connection delay, queuing delay, and throughput starts approximately at $100 \mathrm{~s}$. The duration of application has been set to end of profile.

6.2. Observations. Figure 3 illustrates the connection delay for the four-bandwidth request (BR) mechanisms with suburban fixed pathloss model. The connection delay remains constant over the simulation run for the four BR mechanisms.

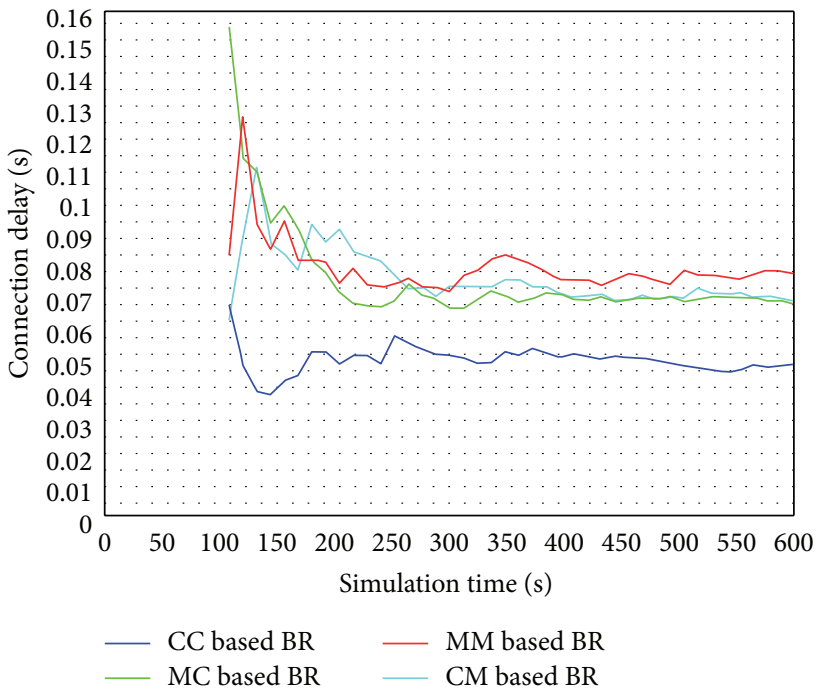

FIGURE 4: Connection delay as a function of simulation period for vehicular pathloss model.

It can be perceived that the connection delay is less with $\mathrm{MC}$ based BR and high with CM based BR. With MC based BR, the connection delay is smaller by $6 \%, 22.99 \%$, and $9.1 \%$ than $\mathrm{CC}, \mathrm{CM}$, and MM based BR, respectively. With an average delay spread of $35 \mathrm{~ns}$ in suburban pathloss model, the main cause of transmission failure at the RS is due its detection threshold (failure due to detection of CDMA codes). The MS with code based BR between MS and RS suffers from large transmission failure than message based BR. The connection delay curves with vehicular pathloss models are shown in Figure 4. As different from previous scenario (suburban 


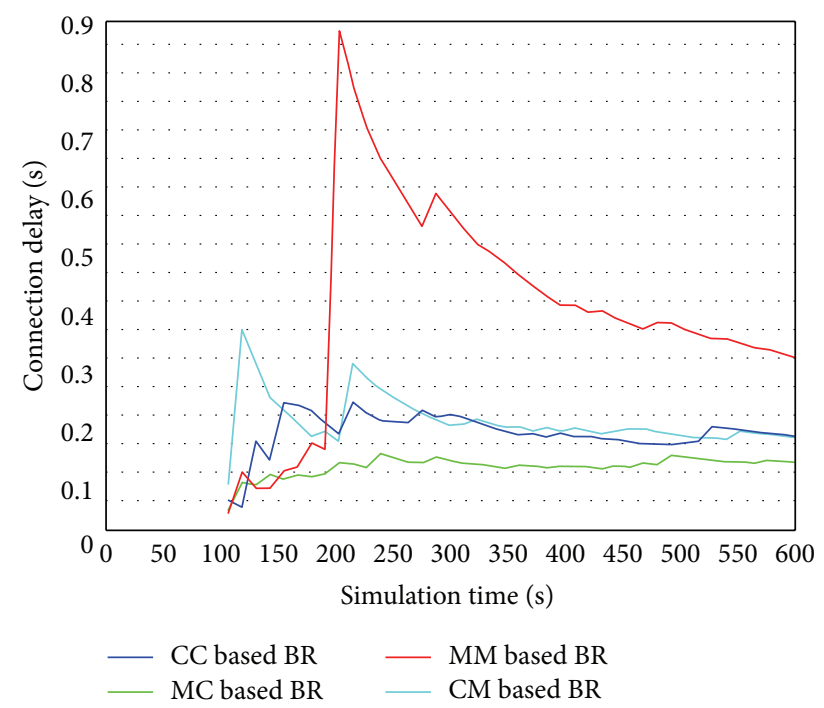

FIGURE 5: Connection delay as a function of simulation period for outdoor to indoor and pedestrian pathloss model.

pathloss model), the CC based BR performs better than other three BR mechanisms. With a delay spread of $370 \mathrm{~ns}$ in vehicular pathloss model, the transmission failure by collision due to contention dominates other failures, namely, channel error, unavailability of bandwidth, and failure due to detection of CDMA codes. Hence, the code based BR at both hops is preferred than message based BR. With CC based BR, the connection delay is cut down by $21.9 \%, 29.79 \%$, and $30.15 \%$ than MC, CM, and MM based BR, respectively.

Figure 5 shows the connection delay curves with outdoor to indoor and pedestrian pathloss model. The performance of BR mechanisms for outdoor to indoor and pedestrian pathloss model lies between their performance for suburban fixed and vehicular pathloss model. As in suburban fixed, the performance of MC based BR performs better than other three BR mechanisms. In suburban fixed, the MM based BR performs better than CM based BR. However, with outdoor to indoor and pedestrian pathloss model, the CM based BR performs better than MM based BR, and further it overlaps with CC based BR. With delay spread of $45 \mathrm{~ns}$ for outdoor to indoor and pedestrian pathloss model, the main cause of transmission failure is due to detection threshold than collision due to contention and unavailability of bandwidth. With MC based BR, the connection delay is lesser by $30.16 \%$, $32.79 \%$, and $69.06 \%$ than CC, CM, and MM based BR, respectively.

The queuing delay curves with suburban fixed pathloss model are shown in Figure 6. Queuing delay is the time between packets that arrive at the queue and those transmitted. The queuing delay mainly depends on the MAC protocol used by the stations (MS and RS). Since the queuing delay is proportional to the buffer size, the lesser the queuing delay, the more data can be buffered at the RS. In simulation, the buffer size of RS has been set to 128 kbytes. With reduced connection delay, the number of retransmission attempts (due to transmission failure) is reduced and hence the

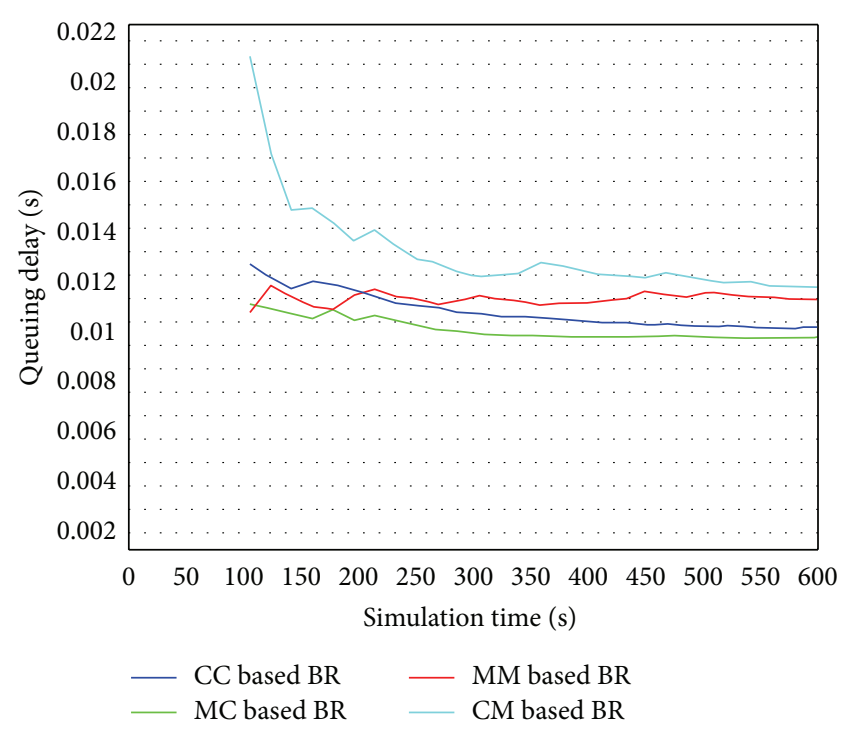

FIGURE 6: Queuing delay as a function of simulation period for suburban fixed pathloss model.

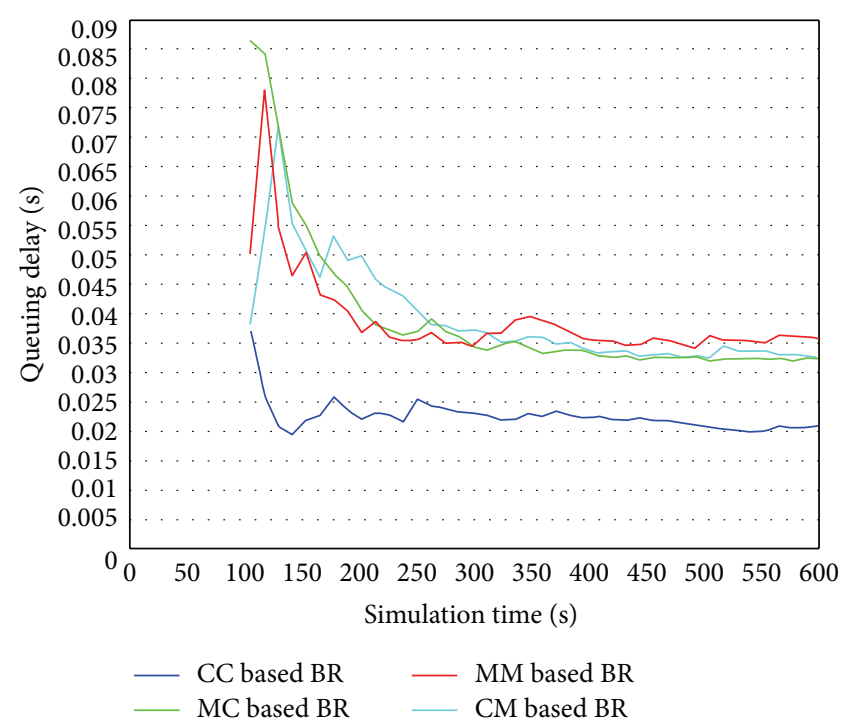

FIgURE 7: Queuing delay as a function of simulation period for vehicular pathloss model.

queuing delay with MC based BR is also less than other three bandwidth request mechanisms. The queuing delay with MC based BR is lessened by $8.47 \%, 10.85 \%$, and $29.16 \%$ than CC, $\mathrm{MM}$, and CM based BR, respectively.

The queuing delay with vehicular pathloss model is shown in Figure 7. Since the connection delay is lesser with CC based BR under vehicular pathloss model, the queuing delay is also reduced. With CC based BR, the queuing delay is lower by $33.35 \%, 42.3 \%$, and $38.52 \%$ than MC, CM, and MM based BR, respectively. Figure 8 illustrates the queuing delay with outdoor to indoor and pedestrian pathloss model. Since the connection delay is lesser with MC based BR under outdoor to indoor and pedestrian pathloss model, the queuing delay is also reduced. With MC based BR, the 


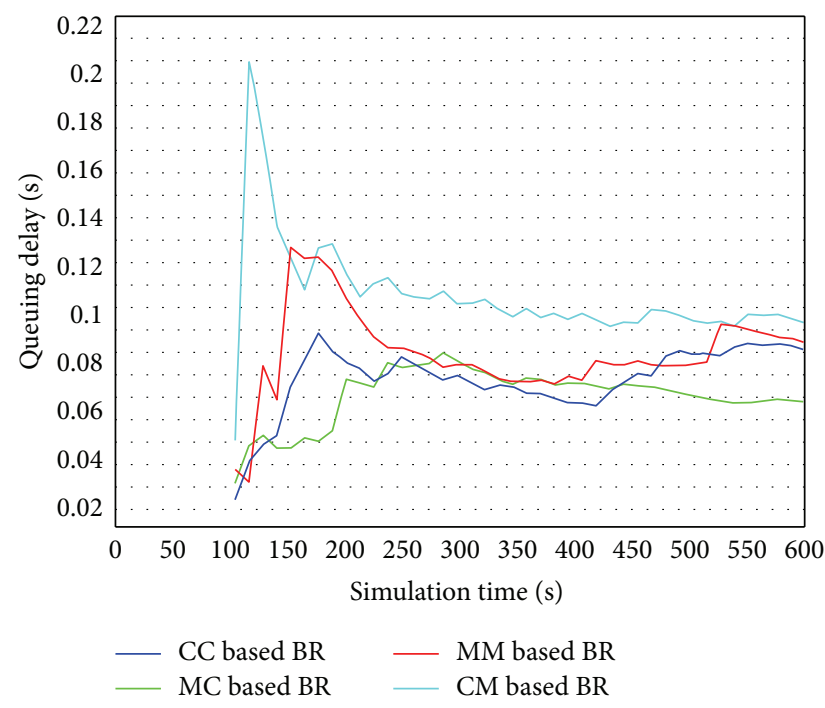

FIGURE 8: Queuing delay as a function of simulation period for outdoor to indoor and pedestrian pathloss model.

queuing delay is smaller by $12.36 \%, 39.12 \%$, and $23.27 \%$ than CC, CM, and MM based BR, respectively.

The performance curves for throughput for the four bandwidth request mechanisms are shown in Figure 9 with suburban fixed pathloss model. The throughput increases with increase in the simulation time that is directly proportional to the increase in the number of successful bandwidth request by the MSs. The throughput of the network depends on number of factors, namely, block error rate (BLER), average uplink packet drop, connection delay, and queuing delay. The BLER is given by the number of bandwidth request failure to the total number of request made by the RS and MS.

The packet drop at the BS can be caused by a number of factors including signal degradation over the network due to multipath fading, collision due contention, channel congestion, signal to noise ratio, unavailability of bandwidth, and distance between the transceivers. Further, with contention based BR at each hop, there is high possibility of packet being dropped at RSs, and hence the retransmission within the network increases. With reduced connection delay and queuing delay, the throughput for MC based $\mathrm{BR}$ is improved by $3.36 \%, 5.62 \%$, and $11.92 \%$ than CC, CM, and MM based $\mathrm{BR}$, respectively.

The throughput curves with vehicular pathloss model are shown in Figure 10. The throughput for CC based BR is improved by $4.18 \%, 14.99 \%$, and $8.13 \%$ than MC, CM, and MM based BR, respectively. The throughput with outdoor to indoor and pedestrian pathloss model is shown in Figure 11. The throughput for MC BR is improved by $12.32 \%, 54.80 \%$, and $33.42 \%$ than CC, CM, and MM BR, respectively. The connection delay, queuing delay, and throughput of the four BR mechanisms under three pathloss models are shown in Table 3. From Table 3 it is found that the CC BR is appropriate for pathloss with high delay spread (vehicular environment), and MC BR is preferred with less and moderate delay spread,

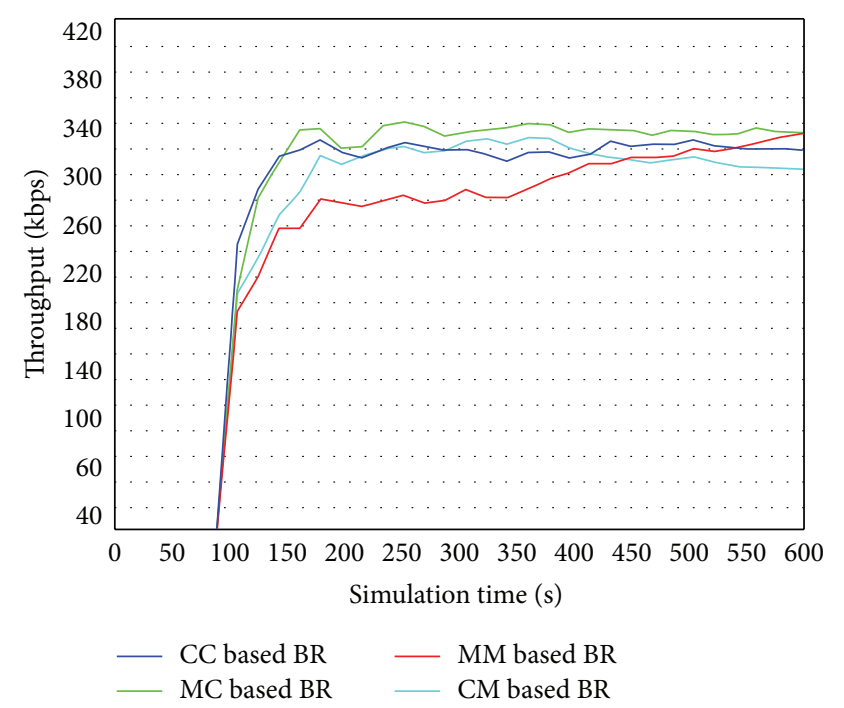

FIGURE 9: Network throughput as a function of simulation period for suburban fixed pathloss model.

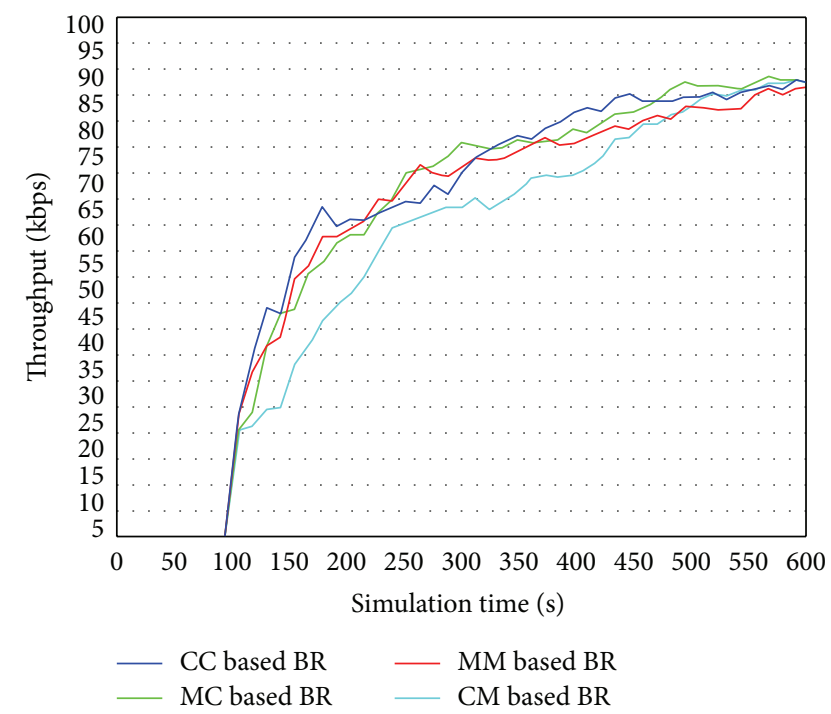

FIGURE 10: Network throughput as a function of simulation period for vehicular pathloss model.

namely, suburban fixed and outdoor to indoor/pedestrian environments.

\section{Conclusion}

In this paper, the performance of contention based bandwidth request for WiMAX relay networks under ITU-T vehicular model has been investigated. Simulations validate the contention based BR mechanism in terms of network throughput, connection delay, and queuing delay. The CC BR performs better than MM, MC, and CM BR with vehicular pathloss model. With the CC based BR, the throughput is improved by $4.18 \%$, the connection delay is reduced by $21.9 \%$, and the queuing delay is reduced by $33.35 \%$ compared to 
TABLE 3: Comparison of contention based bandwidth request mechanisms under various ITU-R pathloss models.

\begin{tabular}{lccccccccc}
\hline \multirow{2}{*}{ Types } & \multicolumn{3}{c}{ Suburban fixed } & \multicolumn{3}{c}{ Vehicular model } & \multicolumn{3}{c}{ Outdoor to indoor and pedestrian } \\
& $\begin{array}{c}\text { Connection } \\
\text { delay }(\mathrm{s})\end{array}$ & $\begin{array}{c}\text { Queuing } \\
\text { delay }(\mathrm{s})\end{array}$ & $\begin{array}{c}\text { Throughput } \\
(\mathrm{kbps})\end{array}$ & $\begin{array}{c}\text { Connection } \\
\text { delay }(\mathrm{s})\end{array}$ & $\begin{array}{c}\text { Queuing } \\
\text { delay }(\mathrm{s})\end{array}$ & $\begin{array}{c}\text { Throughput } \\
(\mathrm{kbps})\end{array}$ & $\begin{array}{c}\text { Connection } \\
\text { delay }(\mathrm{s})\end{array}$ & $\begin{array}{c}\text { Queuing } \\
\text { delay }(\mathrm{s})\end{array}$ & $\begin{array}{c}\text { Throughput } \\
(\mathrm{kbps})\end{array}$ \\
\hline Message-message (MM) & 0.02425 & 0.01090 & 251.67 & 0.07760 & 0.03660 & 61.50 & 0.3367 & 0.07233 & 603.33 \\
Message-code (MC) & $\mathbf{0 . 0 2 2 0 5}$ & $\mathbf{0 . 0 0 9 7 1}$ & $\mathbf{2 8 1 . 6 7}$ & 0.06940 & 0.03376 & 63.83 & $\mathbf{0 . 1 0 4 2}$ & $\mathbf{0 . 0 5 5 5}$ & $\mathbf{8 0 5 . 0 0}$ \\
Code-message (CM) & 0.02863 & 0.01372 & 266.67 & 0.07720 & 0.03900 & 57.83 & 0.1550 & 0.09117 & 520.00 \\
Code-code (CC) & 0.02345 & 0.01062 & 272.50 & $\mathbf{0 . 0 5 4 2 0}$ & $\mathbf{0 . 0 2 2 5 0}$ & $\mathbf{6 6 . 5 0}$ & 0.1492 & 0.06333 & 716.67 \\
\hline
\end{tabular}

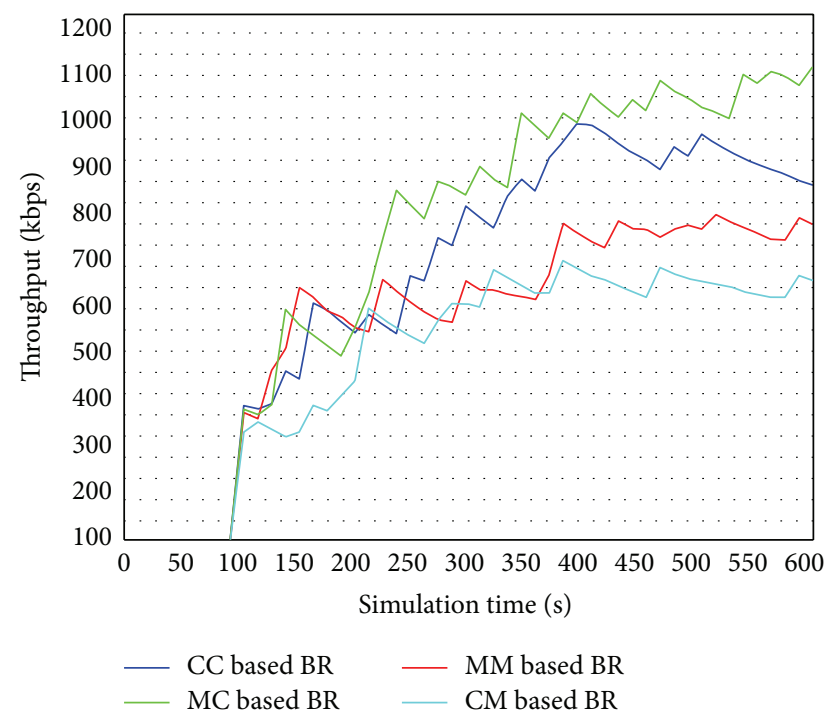

FIGURE 11: Network throughput as a function of simulation period for outdoor to indoor and pedestrian pathloss model.

MC based BR under vehicular pathloss model. Conversely, the MC BR performs better than MM, CC, and CM BR with suburban fixed and outdoor to indoor/pedestrian environments. With MC based BR, the throughput is improved by $3.36 \%$, the connection delay is reduced by $6 \%$, and the queuing delay is reduced by $8.47 \%$ compared to CC based BR under suburban fixed pathloss model. Also with MC based $\mathrm{BR}$, the throughput is improved by $12.32 \%$, the connection delay is reduced by $30.16 \%$, and the queuing delay is reduced by $12.36 \%$ compared to CC based BR under outdoor to indoor/pedestrian environment pathloss model. Hence, when the delay spread is high (450 ns), the CC BR is preferred at the MS, and for lesser range of delay spread (35 ns to $45 \mathrm{~ns}$ ), the MS can carry out MC BR.

\section{Acknowledgments}

This work is supported in part by Department of Science and Technology (DST), Ministry of Science and Technology (MST), India, under the Innovation in Science Pursuit for Inspired Research (INSPIRE) support program of AORC (Assured Opportunity for Research Career).

\section{References}

[1] IEEE Standard for Local and Metropolitan Area Networks Part 16: Air Interface for Broadband Wireless Access Systems, pp. C12004, May 2009.

[2] W. H. Liao, K. P. Shih, C. Liu, A. K. Dubey, S. Arora, and S. P. Kedia, "An efficient scheduling algorithm for radio resource reuse in IEEE 802.16j multi-hop relay networks," Computers and Electrical Engineering, vol. 37, no. 4, pp. 511-525, 2011.

[3] F. M. Chang, I. P. Hsieh, and S. J. Kao, "An efficient uplink scheduling mechanism with enabling multi-device transmission and maximum latency fulfillment in IEEE 802. 16j networks," in Proceedings of 6th International Conference on Computer Science \& Education (ICCSE '11), pp. 1410-1415, August 2011.

[4] M. Salem, A. Adinoyi, H. Yanikomeroglu, and Y. D. Kim, "Radio resource management in OFDMA-based cellular networks enhanced with fixed and nomadic relays," in Proceedings of the IEEE Wireless Communications and Networking Conference (WCNC '10), pp. 1-6, April 2010.

[5] S. M. Kim, S. H. Kim, S. H. Yoon, and D. K. Sung, "A hybrid radio resource management scheme for uplink relay-based cellular networks," in Proceedings of the IEEE 20th Personal, Indoor and Mobile Radio Communications Symposium (PIMRC '09), pp. 1873-1877, September 2009.

[6] Z. Abichar, A. E. Kamal, and J. M. Chang, "Planning of relay station locations in IEEE 802.16 (WiMAX) networks," in IEEE Wireless Communications and Networking Conference (WCNC '10), April 2010.

[7] V. Genc, S. Murphy, and J. Murphy, "Performance analysis of transparent relays in 802.16j MMR networks," in Proceedings of the 6th Intl. Symposium on Modeling and Optimization in Mobile, Ad Hoc, and Wireless Networks (Wiopt'08), pp. 273-281, April 2008.

[8] F. Wang, A. Ghosh, C. Sankaran, P. J. Fleming, F. Hsieh, and S. J. Benes, "Mobile WiMAX systems: performance and evolution," IEEE Communications Magazine, vol. 46, no. 10, pp. 41-49, 2008.

[9] J. Zhang, G. Liu, F. Zhang, L. Tian, N. Sheng, and P. Zhang, "Advanced international communications," IEEE Vehicular Technology Magazine, vol. 6, no. 2, pp. 92-100, 2011.

[10] K. Bakowski and K. Wesolowski, "Change the channel," IEEE Vehicular Technology Magazine, vol. 6, no. 2, pp. 82-91, 2011.

[11] N. Lee, Y. Choi, S. Lee, and N. Kim, "A new CDMA-based bandwidth request method for IEEE 802.16 OFDMA/TDD systems," IEEE Communications Letters, vol. 14, no. 2, pp. 124126, 2010.

[12] C. C. Chong, F. Watanabe, H. Inamura, K. Kitao, and T. Imai, "Performance comparison of the 3GPP/3GPP2 SCM and ITUR IMT-advanced MIMO channel models," in Proceedings of the 
IEEE 20th Personal, Indoor and Mobile Radio Communications Symposium (PIMRC '09), September 2009.

[13] P. Begovic, N. Behlilovic, and E. Avdic, "A novel approach for evaluating applicability of existing empirical propagation models to coverage planning in $3.5 \mathrm{GHz}$ WiMAX systems," in Proceedings of 18th International Conference on Systems, Signals and Image Processing (IWSSIP '11), pp. 1-9, June 2011.

[14] C. Ide, B. Dusza, and C. Wietfeld, "Mobile WiMAX performance measurements with focus on different QoS targets," in Proceedings of 18th IEEE Workshop on Local \& Metropolitan Area Networks (LANMAN '11), pp. 1-6, October 2011.

[15] D. De Luca, F. Fiano, F. Mazzenga, C. Monti, S. Ridolfi, and F. Vallone, "Outdoor path loss models for IEEE 802.16 in suburban and campus-like environments," in Proceedings of the IEEE International Conference on Communications (ICC '07), pp. 4902-4906, June 2007.

[16] J. De Bruyne, W. Joseph, L. Verloock, and L. Martens, "Measurements and evaluation of the network performance of a fixed WiMAX system in a suburban environment," in Proceedings of the IEEE International Symposium on Wireless Communication Systems (ISWCS '08), pp. 98-102, October 2008.

[17] J. W. So, "Performance analysis of VoIP services in the IEEE 802.16e OFDMA system with inband signaling," IEEE Transactions on Vehicular Technology, vol. 57, no. 3, pp. 1876-1886, 2008.

[18] F. E. Ismael, S. K. Syed Yusof, and N. Fisal, "Bandwidth grant algorithm for delay reduction in IEEE 802.16j MMR WiMAX networks," International Review on Computers and Software, vol. 5, no. 2, pp. 242-248, 2010.

[19] B. Upase and M. Hunukumbure, "Dimensioning and cost analysis of multihop relay-enabled WiMAX networks," Fujitsu Scientific and Technical Journal, vol. 44, no. 3, pp. 303-317, 2008.

[20] K. C. Chu and T. C. Huang, "A novel bandwidth request mechanism for IEEE 802.16j networks," Tamkang Journal of Science and Engineering, vol. 13, no. 1, pp. 71-78, 2010.

[21] D. Niyato, E. Hossain, D. I. Kim, and Z. Han, "Relay-centric radio resource management and network planning in IEEE 802.16j mobile multihop relay networks," IEEE Transactions on Wireless Communications, vol. 8, no. 12, pp. 6115-6125, 2009.

[22] P. Mach and R. Bestak, "Analysis and performance evaluation of IEEE 802.16 enhanced with decentrally controlled relays," in Proceedings of the 16th International Conference on Systems, Signals and Image Processing (IWSSIP '09), pp. 1-6, June 2009.

[23] Z. Becvar and P. MacH, "Reduction of scanning reporting overhead in IEEE 802.16 networks with relays," in Proceedings of the 9th International Conference on Networks (ICN '10), pp. 109-114, April 2010.

[24] J. Zhang, S. Feng, W. Ye, and H. Zhuang, "Reducing signaling overhead and latency of $802.16 j$ service flow management," in Proceedings of the International Conference on Wireless Communications, Networking and Mobile Computing (WiCOM '08), October 2008.

[25] Y. T. Mai, C. C. Yang, J. Y. Chen, and K. Y. Chen, "A zonebased bandwidth allocation protocol in WiMAX multi-hop relay networks," in Proceedings of the International Conference on Information Networking (ICOIN '11), pp. 257-261, January 2011.

[26] E. Calvo, J. Vidal, and J. R. Fonollosa, "Optimal resource allocation in relay-assisted cellular networks with partial CSI," IEEE Transactions on Signal Processing, vol. 57, no. 7, pp. 28092823, 2009.
[27] Y. P. Fallah, F. Agharebparast, M. R. Minhas, H. M. Alnuweiri, and V. C. M. Leung, "Analytical modeling of contentionbased bandwidth request mechanism in IEEE 802.16 wireless networks," IEEE Transactions on Vehicular Technology, vol. 57, no. 5, pp. 3094-3107, 2008.

[28] Q. Ni, L. Hu, A. Vinel, Y. Xiao, and M. Hadjinicolaou, "Performance analysis of contention based bandwidth request mechanisms in WiMAX networks," IEEE Systems Journal, vol. 4, no. 4, pp. 477-486, 2010.

[29] D. Chuck, K. Y. Chen, and J. M. Chang, "A comprehensive analysis of bandwidth request mechanisms in IEEE 802.16 networks," IEEE Transactions on Vehicular Technology, vol. 59, no. 4, pp. 2046-2056, 2010.

[30] R. Anbazhagan and N. Rangaswamy, "Contention resolution with EIED backoff for bandwidth request in IEEE 802.16 networks," Elsevier International Journal of Electronics and Communications, vol. 67, no. 1, pp. 40-44, 2013.

[31] International telecommunications Union-Recommendations (ITU-R), "Guidelines for evaluation of radio interface technologies for IMT-Advanced," ITU-R Report M. 2135, Geneva, Switzerland, 2008. 

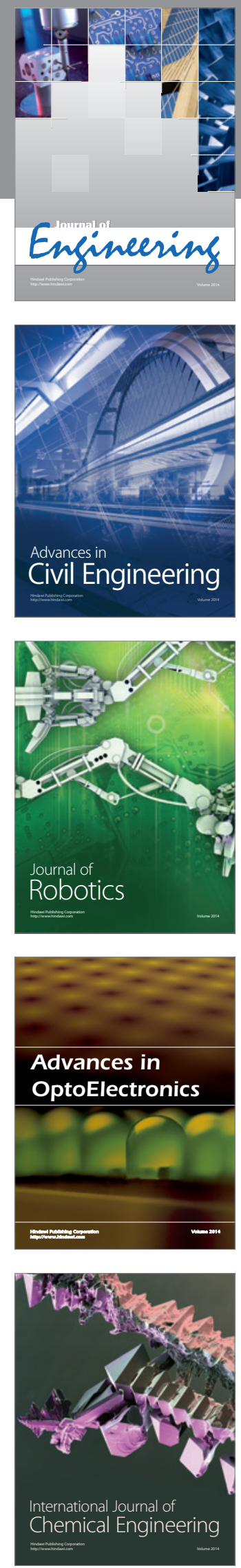

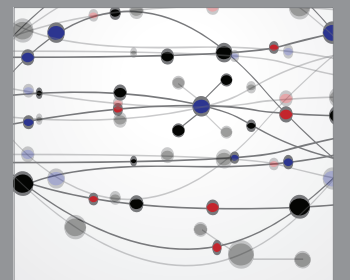

The Scientific World Journal
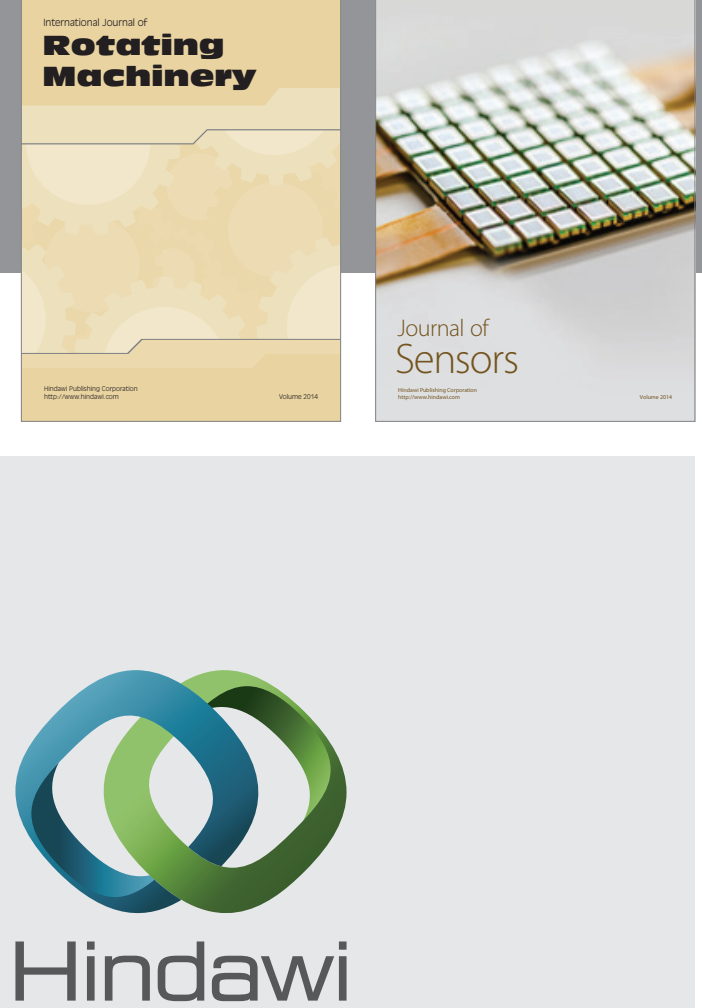

Submit your manuscripts at http://www.hindawi.com
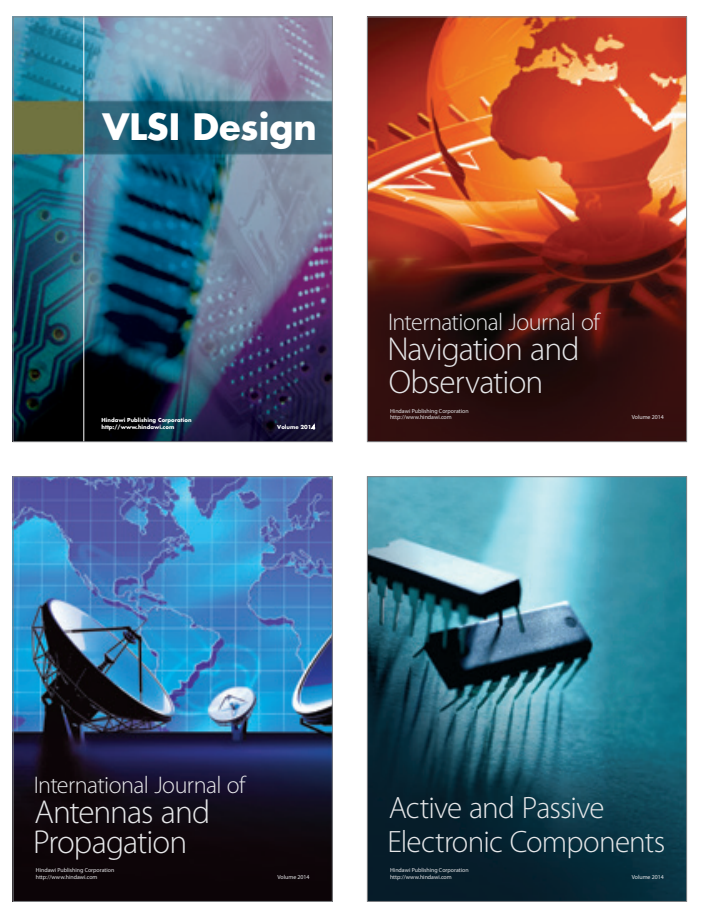
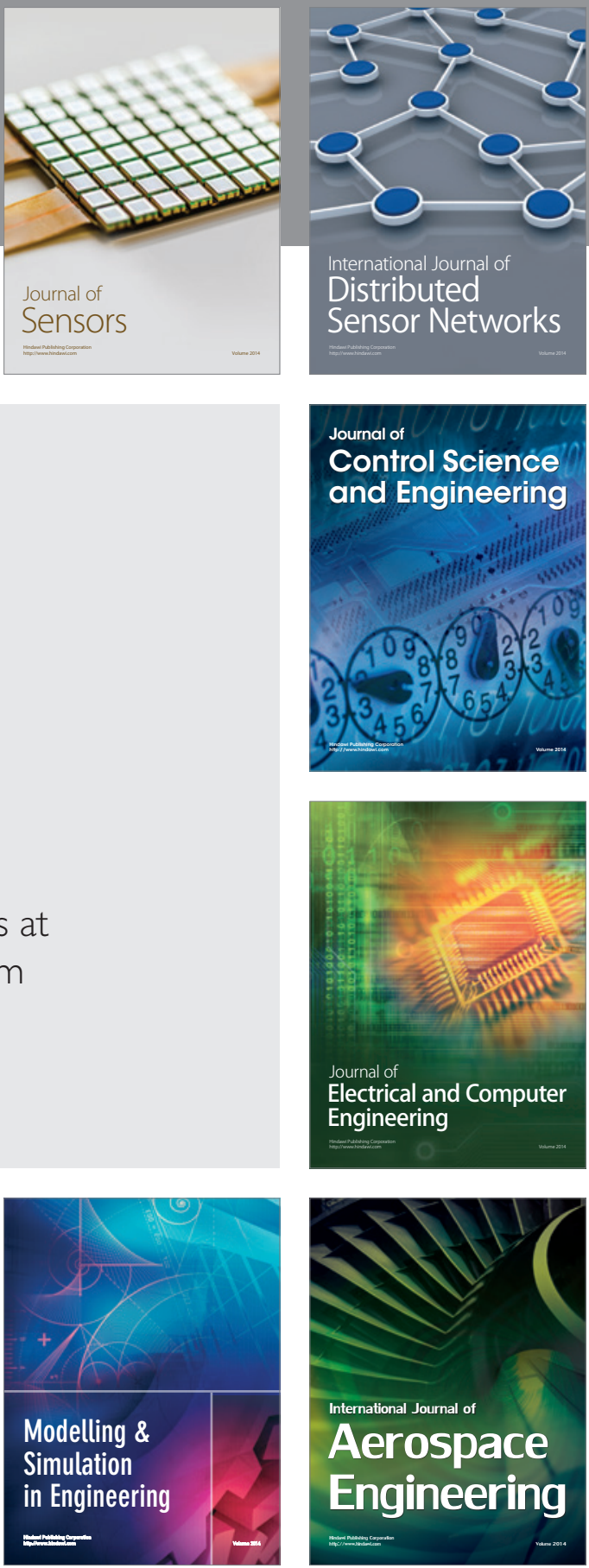

Journal of

Control Science

and Engineering
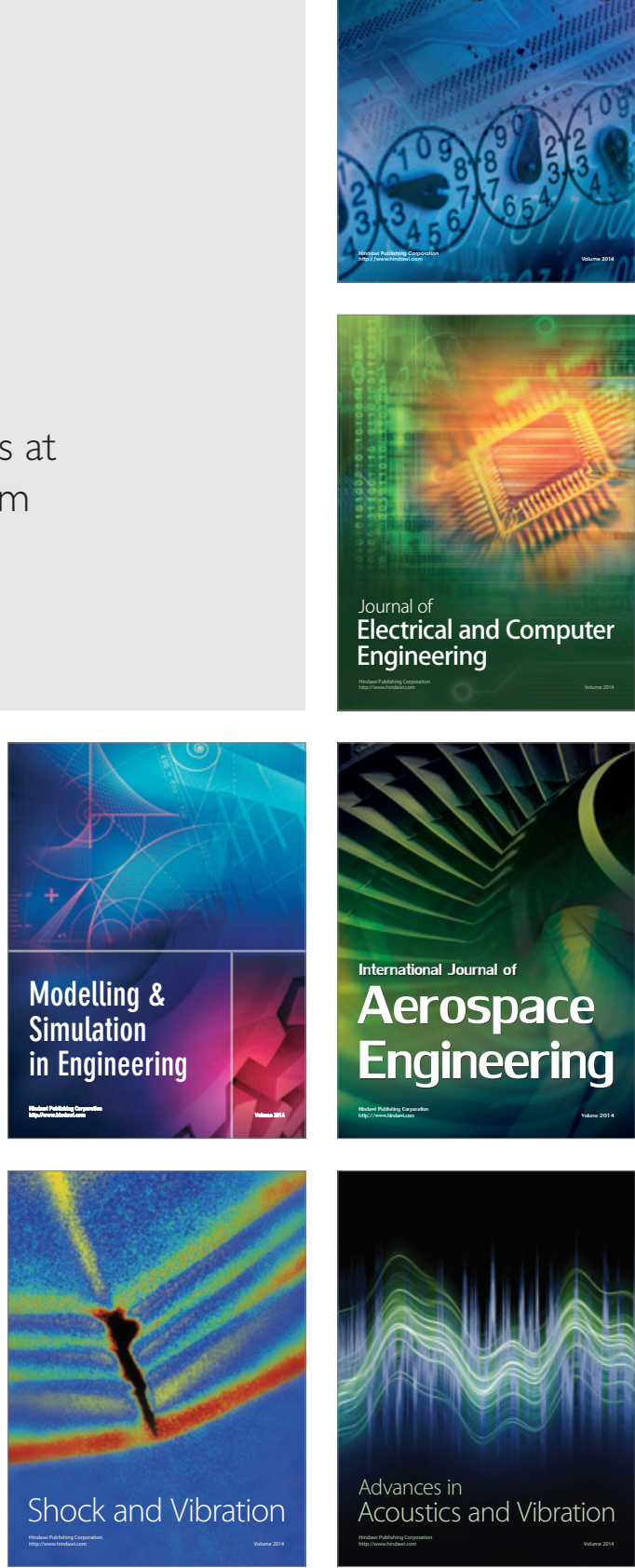